\title{
Review of: "Blocking a dead-end assembly pathway creates a point of regulation for the DEAD-box ATPase Has1 and prevents platform misassembly"
}

\author{
William Blalock ${ }^{1}$ \\ 1 Italian National Research Council
}

Potential competing interests: The author(s) declared that no potential competing interests exist.

Summary: The manuscript by Liu et al., details and characterizes a critical point of control in eukaryotic ribosome biogenesis. The authors detail the role of the Rrp5-Rrp36 interaction for normal ribosome biogenesis of the $40 \mathrm{~S}$ subunit and the requirement for the DEAD-box ATPase, Has 1, to catalyze the disruption of the Rrp5-Rrp36 interaction in the assembling body in order to allow Rrp5 repositioning to the platform. The importance of Has 1 in this step was also demonstrated in assembly of late-binding factors due to its influence on the UtpB switch. All-in-all the manuscript is well written and defines a basic biological process necessary for proper ribosome biogenesis and subsequent protein synthesis. There are several issues that should be addressed by the authors in order for the reader to have a more critical perspective of the data as well as some minor suggestions.

1. Seeing the importance of the Rrp5 and Rrp36 interaction in this process, it might be worthwhile to include this in the title.

2. At the bottom of page 4, "either" is used without an "or". The "or " is the following sentence that begins with "Alternatively".

3. The data discussed in the middle paragraph on page 8 is placed in supplemental, but as this data is key to the role of Has 1, I would include it as part of a regular figure.

4. On page 10, the 13th and 14th line from the bottom of the page beginning, "Three biological replicates....", would be better placed in the figure legend or methods.

5. On page 11, the first and second paragraphs contain information better suited to the Discussion.

6. On page 12, middle paragraph (Rps 1 incorporation.....) the next to the last line contains an additional "that".

7. In general, the figures need to be more organized to the text. On several occasions figures contain several panels that are discussed and other panels that are not discussed until much later. A prime example is the data presented in Figure 4 where certain data in Figure 4 is not discussed until after a discussion of Figure 5.

8. At the bottom of page 19 and the top of page 20, close the brackets after $2 \mathrm{mM} \mathrm{BME}$.

9. From the author's description, it appears that in the absence of Rrp36, Rps5 has the ability to 
misassemble resulting in dead-end products, but also evidently, as observed with the delta S1 mutant of Rrp5, able to form a partially functioning ribosome since ribosome biogenesis and cell growth does not come to a complete stop. What would be or could be the advantage of having this ability? Or is the effect a dominant-negative effect where endogenous Rrp5 and Rrp36 can still partially compensate? In other words, if proper assembly of Rrp5 is absolutely required for ribosome biogenesis, evolutionarily speaking why would Rrp5 retain the ability to even form dead-end products rather than form no product at all? Do the dead-end products have any additional physiological role? This question relates to findings reported in Figure 3 where delta S1 Rrp5 enhanced stop codon read-throughs.

10. For figures using fractions from sucrose gradients, it would be better to included in the figure legend the description of the fractions as stated in the Materials and Methods as well as label the lanes as fraction \#.

11. In Figure 1A, a MBP control is necessary to exclude any non-specific binding. Ratios of input vs captured should be included for comparison between the pull-downs. It would also help at this point to refer the reader to the schematic diagram in Supplemental figure S1C.

12. Figure 4 legend, the last sentence of $C$ is odd ("averages from at from"). Also D refers to blue, red and green boxes; while only red boxes are visible in the figure.

13. In Figure 5 panel A, the authors need to include a HA only control.

14. Due to the analysis of fractions in western blot between wild-type and mutants and the limited lanes on gels, fractions of the same experimental type are run on different gels and blotted on separate membranes. This approach makes it extremely hard to compare test groups as loading, transfer, immunoprobing and exposure are not controlled for.

15. The authors should include all raw and analyzed data (\# of fragments, \# of unique fragments, \% coverage, etc.) for mass spec as supplemental data to support data shown in the bar charts presented in the manuscript. 